\title{
DNA repair and indels boost errors
}

Mutation rates vary with genomic locus and cellular context, but there are many unanswered questions regarding when, where and how elevated mutation rates occur. Two papers now implicate DNA repair in increased local mutation rates, and the results may influence future studies of genetic variation and tumorigenesis.

Mitotic gene conversion is a mechanism for repairing DNA double-strand breaks (DSBs) in which a homologous sequence (donor) is used as a template for repair. Hicks and colleagues took advantage of the gene conversion that is a normal part of mating-type (MAT) switching in Saccharomyces cerevisiae to screen for errors generated by this repair process. They inserted a gene (URA3)

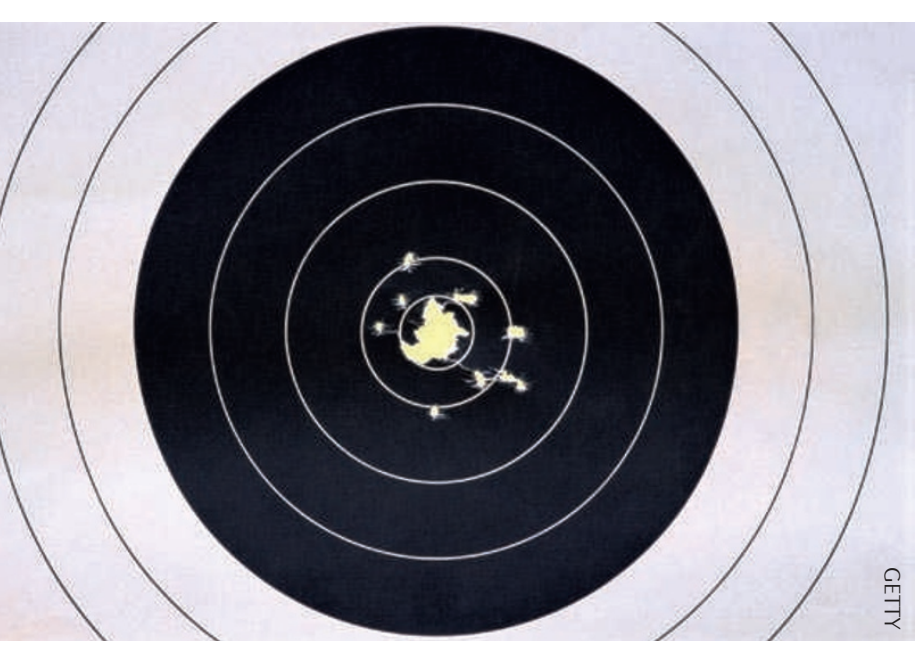

into the normal donor sequence that enabled them to chemically select cells in which mutations had arisen during gene conversion. Strikingly, they found that the mutation rate associated with gene conversion was 1,400 times higher than the rate of spontaneous mutation in the same genomic region.

Hicks et al. showed that the majority of mutations were singlebase-pair substitutions, but a high proportion were one-base-pair deletions and complex mutations that are probably caused by templateswitching during gene conversion. Surprisingly, the normally highfidelity DNA polymerase- $\delta$ seems to cause these template switches. This finding suggests that gene conversion is less processive than the DNA synthesis that takes place during $S$ phase. Furthermore, the authors suggest that some mutations required for carcinogenesis could result from gene conversion being used to repair the increased numbers of DSBs triggered by activated oncogenes.

In another study, De and Babu analysed the occurrence of singlenucleotide substitutions (for example, SNPs) near to structural alterations (for example, indels) in sequences separated by different lengths of time: the reference human and chimpanzee genomes, the reference human genome and human personal genomes, and human cancer and somatic cell genomes. They found that, irrespective of the time separating the genomes, there are increased single-nucleotide changes in the neighbourhood of structural changes. Various DNA-repair mechanisms could be involved in forming indels, and the authors suggest that errors introduced during repair, perhaps by low-fidelity polymerases, are a cause of the nearby single-nucleotide changes. De and Babu note that this relationship between indels and SNPs suggests that mutations of different sizes in the vicinity of a marker SNP should be considered when interpreting results from genome-wide association studies.

Both of these studies provide evidence that mechanisms involved in maintenance of global genomic integrity can cause damage at a local level. Therefore, useful insights might be gained from considering observations made in the context of disease genomics or population studies in the light of elevated mutation rates resulting from repair pathways.

\section{Mary Muers}

ORIGINAL RESEARCH PAPERS Hicks, W. M., Kim, M. \& Haber, J. E. Increased mutagenesis and unique mutation signature associated with mitotic gene conversion. Science 329, 82-85 (2010)|De, S. \& Babu, M. M. A time-invariant principle of genome evolution. Proc. Natl Acad. Sci USA 6 Jul 2010 (doi:10.1073/pnas.0914454107) FURTHER READING Hastings, P. J.et al.

Mechanisms of change in gene copy numbers. Nature Rev. Genet. 10, 551-564 (2009) 